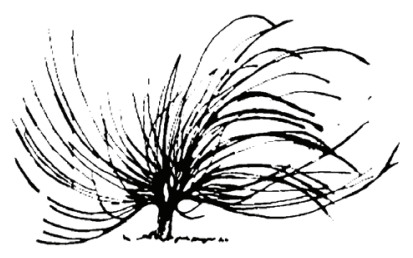

\title{
Sobre la necesidad de la enseñanza directa del vocabulario en los niveles primario y secundario del sistema educativo argentino
}

\author{
Martín Gonzalo Zapico ${ }^{1}$ \\ Universidad Nacional de Mar del Plata-Instituto Nacional de \\ Formación Docente Continua \\ Mar del Plata, Buenos Aires, Argentina \\ athenspierre@gmail.com
}

\begin{abstract}
Resumen
En el sistema educativo argentino hoy se vive una situación de crisis, que se hace evidente cuando los egresados de secundaria deben afrontar una carrera universitaria: no tienen las competencias básicas de lectura y escritura, lo cual redunda en una alta tasa de deserción en el primer año. Este estado de situación, entre otras cosas, puede ser atribuido a un enfoque que optó por dejar de lado la enseñanza directa del vocabulario, postulando que el aprendizaje de este puede darse de manera indirecta. En función de esto proponemos que la enseñanza directa del vocabulario es necesaria para promover no solo un mejor desempeño académico, sino una mejor calidad de vida
\end{abstract}

\section{(9) $(1) \Theta(\Theta$}

Recibido: 18 de julio de 2016-Aprobado: 3 de octubre de 2017

http://dx.doi.org/10.15359/rep.12-2.2

1 Profesor en Letras, recibido en la Universidad Nacional de Mar del Plata. Actualmente se desempeña como docente-investigador en las cátedras de Introducción a los Estudios Literarios y Teoría Literaria. Ha publicado variedades de artículos en las áreas de lingüística, educación y psicología, y asistido a numerosos congresos de dichas disciplinas. 
en general. Para argumentar esta posición, acudiremos a la opinión de docentes, expertos en el tema, estudios teóricos y experiencias prácticas, poniendo en evidencia que es urgente un retorno a estas prácticas para promover el desarrollo del vocabulario en un mundo que tiende cada vez más a la economía del lenguaje.

Palabras clave: enseñanza, aprendizaje, vocabulario, desempeño académico.

\begin{abstract}
In the Argentine educational system today we live a crisis situation, which becomes evident when high school graduates must face a college major: They do not have the basic skills of reading and writing, which results in a high dropout rate in the first year. This state of affairs, among other things, can be attributed to an approach that chose to ignore the direct teaching of vocabulary learning, postulating that it can be indirectly learned. On this basis we propose that the direct teaching of vocabulary is necessary to promote not only a better academic performance but a better quality of life in general. To argue this position, we will request the opinion of teachers, experts in the field, theoretical studies and practical experience, highlighting the urgency of a return to these practices to promote vocabulary development in a world that tends increasingly to the economy of language
\end{abstract}

Keywords: teaching, learning, vocabulary, academic performance.

\title{
Introducción
}

El siguiente ensayo surge a partir de una problemática concreta, que docentes de distintos niveles del sistema educativo en la Argentina debemos afrontar día a día: la cada vez más escasa competencia léxica que tienen los alumnos de distintos niveles educativos. Este estado de situación puede ser documentado de diversas formas: la charla con colegas de distintas instituciones, estudios que revelan las profundas deficiencias en prácticas sencillas como la lectura de un texto y 
la expresión escrita (Valeiras, 2009; Laco, Natalia y Ávila, 2010), la enorme proliferación de talleres de escritura en los cursos de ingreso a un sinfín de carreras de nivel superior (Carlino, 2001; Carlino, 2007; Moyano, 2005), entre otras.

Esta problemática, lejos de pertenecer al plano de lo estrictamente académico, llega también a lo social. Un conocido estudio del presidente de la Real Academia Española Pedro Barcia, llevado a cabo en el año 2007 señalaba que en Argentina los jóvenes usan una cantidad promedio de no más de 240 palabras para comunicarse. Hoy, pasados diez años, se observan las consecuencias de dicho estado de situación, y el asunto parece no mejorar. Incluso los autores más críticos con esa cifra (que señalan que no se tienen en cuenta los vocablos creados por las subculturas juveniles y realizan la llamada "crítica a la cantidad") hablan de una innegable "crisis educativa" en materia del lenguaje (Requejo, 2001; Garfias, 2010).

Esto debe ser tomado seriamente, puesto que un vocabulario más acotado no es solo un dato: es menor posibilidad de expresar lo que se siente, menor precisión para dar a conocer nuestras necesidades, menor calidad comunicativa, menor amplitud de pensamiento, y por sobre todas las cosas una menor capacidad de adecuación contextual (recordemos que ese es el objetivo de la enseñanza del lenguaje a lo largo de los sistemas primario y secundario: hablar bien es tener las herramientas para adecuarse lingüísticamente a la gran variedad de escenarios que nos impone la vida en sociedad). Todos estos factores mencionados, cuando no pueden desarrollarse en su totalidad, redundan en una menor calidad de vida.

Quizá lo más extraño es que cuando uno se acerca a los lineamientos generales para la enseñanza de la lengua tanto en el nivel primario como en el secundario, hay indicaciones concretas en torno a la enseñanza del vocabulario (Ministerio de la Nación, 2014). Este es abordado como una competencia necesaria para el desenvolvimiento en una sociedad letrada, y se propone un aprendizaje gradual, continuo y creciente del vocabulario a través de una diversidad de prácticas tanto orales como escritas. Van desde la lectura de toda una diversidad de textos (la inserción del sujeto en la cultura letrada, que implica leer textos literarios, periodísticos, ensayos, notas de opinión, etc), pasan por la escritura (que cada vez debe ser más autónoma y crítica) y se articulan con la oralidad (donde se comunica a un otro aquello que se 
ha trabajado en las otras dos instancias, propiciando el diálogo y el conocimiento construido entre pares).

No obstante, y pese a que estos lineamientos están ya planteados hace años, sigue habiendo vigencia del denominado "modelo comunicativo" para la enseñanza de la lengua. Este modelo, inspirado en la premisa de que el lenguaje es sobre todo una herramienta de comunicación, ha ido dejando de lado progresivamente la enseñanza de la gramática y el vocabulario de manera directa, argumentando que pueden aprenderse de forma indirecta y por medio de la reflexión sobre el uso.

Lo concreto es que en los últimos diez años las tasas de deserción en la universidad por motivos de competencias básicas de lectura comprensiva y redacción han ido en aumento, al punto de registrarse casos de jóvenes que empiezan carreras universitarias sin poder leer fluidamente en voz alta. En una entrevista a Infobae, el prestigioso profesor José Moure declaró, "Para los jóvenes de hoy, el castellano académico es casi un idioma extranjero" (Infobae, 2012) y puso de manifiesto una gran cantidad de datos en torno a los problemas que enfrentan los nuevos alumnos al tener que leer, comprender y trabajar con textos académicos, sea la especialidad que sea.

Es a partir de esta situación que se propone que es necesaria la enseñanza explícita y consciente de vocabulario como contenido concreto en los niveles tanto primario como secundario. Para justificar esta tesis se hablará primero de las razones que creemos han llevado al fracaso del modelo comunicativo en la enseñanza de la lengua en Argentina, con respecto al tema de la capacidad de leer y escribir. Luego se señalará la importancia de la enseñanza por competencias. Después se mostrará como el vocabulario tiene una correlación directa con el léxico (tanto receptivo como productivo) que es considerado una competencia, dado que posibilita las prácticas de lectura y escritura. Y finalmente se dará apoyo teórico, práctico y científico a la necesidad de enseñar vocabulario de manera directa y como esta enseñanza redundará en un mayor desempeño global del alumnado, en cada una de las instancias del sistema educativo. 


\section{El vocabulario y sus devenires}

Antes de empezar a dar fundamentos de por qué es necesaria la llamada "enseñanza directa" del vocabulario, es pertinente referir a los motivos que llevaron al fracaso del modelo comunicativo de la enseñanza de la lengua, principal responsable del estado de la situación actual.

Quiero matizar la oración anterior, y aclarar que me refiero al principal responsable en el plano de la enseñanza primaria y secundaria estrictamente (es decir, en términos de planes de estudios y lineamientos). Sobre las causas de las deficiencias en las capacidades de lectura y escritura hay mucho escrito, aunque como señala el mismo Moure (Infobae, 2002), los estudios en este campo atribuyen este déficit al abandono de la cultura escrita en pos de la cultura virtual y sus prácticas, que suelen estar muy lejos de las que se realizan en la academia. O sea, hace quince años (cuando el celular estaba en manos de pocos, la internet no era masiva y la televisión era el principal exponente de "la tecnología") aún permanecían las prácticas de ocio relacionadas con la lectura y la escritura, incluso estas eran parte de la vida cotidiana necesariamente. Ni hablar de lo que acontecía hace cuarenta años cuando ante la solicitud de una tarea determinada, el alumno sí o sí debía acudir a la biblioteca, y en el proceso de búsqueda de información, selección y transcripción había un desarrollo de las capacidades de lectura comprensiva y escritura académica.

Marta Marín (2003), en una revisión, se refiere al enfoque comunicativo de la lengua como aquel en el cual predomina la situación comunicativa como condición de excelencia para el aprendizaje de la lengua. Es decir, dado que el lenguaje es una herramienta que utilizamos en la comunicación, es a través de ella que se puede llegar a una adquisición significativa de la lengua, a través de las prácticas del lenguaje que se ponen en juego en el día a día.

Este modelo, señala la autora, vendría a superar el viejo modelo tecnocrático de la enseñanza de la lengua, en el cual el foco estaba puesto en el aprendizaje de abstracciones sin relación alguna con la realidad del alumno, tales como: la gramática en abstracto, el análisis oracional, el uso del diccionario para la adquisición de vocabulario, entre otros. Contra esos preceptos supuestamente obsoletos, se contraponen la llegada a la gramática basándose en el análisis y la reflexión del uso, sacar por completo de los planes de estudio el análisis oracional, desprenderse del diccionario y promover el conocimiento del significado en función 
del contexto, etc. Por último, se habla también de la incorporación de una pluralidad de prácticas. Si antes se privilegiaba el texto escrito de manera casi privativa, en este nuevo enfoque se tratan de forma explícita la oralidad y la expresión como contenidos concretos.

Pese a las posibilidades que señala la autora, ella misma señaló hace trece años el riesgo de que este cambio en el currículo fuera solo "un cambio de etiqueta", y no viniese acompañado de un cambio tanto teórico como práctico real en el aula. Hoy puedo decir, a la luz de los hechos y mi propia experiencia tanto de alumno como de docente de dicho modelo, que no se ha aplicado como se supone debía hacerse; y lo poco que se ha aplicado ha sido simplemente un cambio superfluo en las planificaciones y los contenidos.

No se han capacitado nuevas generaciones de docentes en prácticas del lenguaje, puesto que (como es mi caso) muchos fuimos formados con el enfoque tecnocrático y estamos satisfechos con dicha formación secundaria. Esta resistencia al cambio ha dado como resultado que las cosas varíen a medias: de los currículos se han desterrado los ejercicios referentes al análisis de oraciones y enseñanza directa de vocabulario (cuando la mayor parte de los docentes en actividad son competentes en dichos contenidos); pero en las prácticas los contenidos del enfoque comunicativo no son trabajados desde el enfoque comunicativo. Mis preguntas son: si el enfoque tecnocrático es tan incorrecto, frío e improductivo, ¿por qué los grandes investigadores en lingüística en Argentina y España, así como en otros países de latinoamerica, defienden la enseñanza de la gramática y el vocabulario? ¿En qué modelo fueron ellos formados para llegar a ser quienes son?

Más allá de eso, hay un aspecto que quiero señalar con respecto al enfoque comunicativo incluso en abstracto. La gran apuesta de este enfoque (llegar al análisis metalingüístico a partir del análisis de la situación comunicativa) presupone una capacidad de conceptualización y de análisis abstracto que solo puede otorgar la enseñanza de los conceptos básicos en gramática. El presupuesto de que todos los alumnos son sujetos críticos-reflexivos es peligroso, puesto que para llegar a comprender nociones como "objeto directo", antes debe entenderse la noción de "sustantivo", y para llegar a ella hace falta un repertorio de vocabulario específico que no se puede aprender de manera pasiva por el mero hecho de vivir en una sociedad donde nos comunicamos. La comunicación es necesaria, sí, pero no suficiente. 
En este marco, entra la noción de enseñanza por competencias. Se trata de un giro en la concepción de qué es lo que se debe enseñar. El enfoque basado en la comunicación es precisamente un enfoque basado en competencias, en este caso la comunicativa. Se pasa de enseñar contenidos concretos, a prácticas consistentes en promover capacidades de análisis, síntesis, razonamiento, iniciativa, lectura comprensiva, crítica y todas aquellas que, si bien no son un contenido específico, son herramientas de imprescindible uso para la comprensión de los contenidos (Zapico, 2016).

Una larga cantidad de estudios señalan la bondad y la efectividad de este modelo, tanto en el ámbito internacional, fundamentalmente Europa (Díaz, 2005; García, Bravo, Alvero, Cuello y Sancho, 2005), como a nivel nacional (Aiello y Grandoli, 2012; Vásquez, Rey, Buubé y Cañibano, 2010), donde la tendencia de los currículos nacionales hace foco en la promoción de capacidades fundamentales de lectura comprensiva, juicio crítico, escritura de diversos géneros, entre otras.

La posibilidad de considerar al vocabulario una competencia fundamental, y que por ende merezca un tratamiento de su enseñanza de manera directa, parte de la correlación directa que puede observarse entre el vocabulario y el léxico, tanto receptivo (la capacidad que tiene un sujeto para interpretar textos, sean escritos u orales) como productivo (la capacidad que tiene un sujeto para planificar, organizar y producir textos, sean escritos u orales) (Casso, 2010) De esta manera, una mayor base léxica o lexicón no es un mero aumento cuantitativo, sino también cualitativo. Posibilita no solo decir una mayor cantidad de cosas, sino profundizar y complejizar los enunciados, tal como ha estudiado Chacón Beltrán (2000) en su tesis doctoral donde aborda la incidencia positiva de la enseñanza directa de vocabulario para el aprendizaje de una segunda lengua.

La lengua y, en concreto, la posibilidad de utilizar la expresión verbal como el vocabulario de las diferentes materias de enseñanza es el soporte de todas las materias del currículum escolar (Boehm, 1971; Yuste y García, 1991; Suárez, Moreno y Godoy, 2010). La forma de abordar la enseñanza del vocabulario parece no estar suficientemente sistematizada, olvidándose el proceso cognitivo y de maduración lingüística, así como lo referente a la propia estructura de los campos semánticos (Agustín y Barreras, 2007; Galeote, Peraita y Méndez, 1997; Hasbún, 2005; Mohseni-Far, 2008; Pleyán, 1975; Ur, 1996). De este modo, en 
muchas ocasiones, el niño no adquiere el significado completo de los términos hasta bien avanzada la escolarización.

Una parte importante del fracaso escolar se puede justificar por el desconocimiento de los alumnos de ciertos términos básicos relacionados con las distintas enseñanzas curriculares de la educación infantil (Boehm, 1971; Galve, García y Yuste, 1993). García-Hoz $(1988,1999)$ puso de manifiesto la necesidad de resolver el problema de la integración de los distintos aprendizajes específicos para analizar con posterioridad en qué medida las enseñanzas pueden fundamentar la orientación y formación total del estudiante. Precisamente plantea que para dicha integración se requiere algún elemento que, siendo común a todas las materias, no destruya la singularidad de cada una. Para ello establece la posibilidad de utilizar la expresión verbal y más concretamente el fomento y desarrollo del vocabulario de las diferentes materias de enseñanza como posible vía de integración. Ello es debido, según este investigador, a que en el vocabulario puede encontrarse ese elemento común que necesariamente coexiste con los elementos específicos de cada materia y de ahí la importancia de una enseñanza que tome como base el vocabulario común como elemento curricular integrador.

Entonces, si el vocabulario puede ser considerado como una competencia (en tanto tiene incidencia directa en el desarrollo de habilidades) merecería (a diferencia de lo que propuso y propone el enfoque comunicativo) una enseñanza directa y no indirecta o pasiva en las diversas planificaciones.

Ahora bien, con este panorama, ¿qué garantía tenemos que la enseñanza del vocabulario de manera directa va a dar resultados en este nuevo contexto? Si bien es cierto que la sociedad ha cambiado mucho, hay evidencias de distintos estudios de diferente naturaleza que parecerían ofrecer buenas perspectivas a este tipo de enseñanza.

En un plano teórico se puede encontrar apoyo en el campo de las neurociencias. Específicamente, la teoría neurocognitiva de Sydney Lamb (1999, 2004a, 2004b) que, construyendo hipótesis y enunciados sobre el sistema lingüístico del individuo a partir de los conocimientos que se tienen sobre el cerebro y su relación con el lenguaje, postula que la lengua no es sino un gran conjunto de redes relacionales, donde el "significado" de un lexema no es más que todos aquellos significados que activa su estimulación. Como señala Gil (2009) hay un correlato muy interesante entre esta forma de abordar el lenguaje y las 
implicancias pedagógicas que de ella se derivan. Si estamos ante un conjunto de redes relacionales que se comportan de manera análoga a neuronas, hay fundamentalmente tres procesos posibles para el desarrollo de la red:

- Formación de nuevas conexiones entre nexiones: Posibles relaciones que estaban latentes a partir de un estímulo fuerte empiezan a cobrar actividad.

- Cambio en la fuerza de las conexiones: A través del uso o desuso que se haga de las conexiones, estas podrán aumentar su fuerza o disminuir.

- Cambio en las funciones de umbral: Se define umbral como la mínima cantidad de estímulo que debe recibir una conexión para ser estimulada, así, una conexión que es estimulada frecuentemente pasa a tener un umbral mucho menor que otra que solo es estímulada de manera escasa.

En función de estas posibilidades, la ampliación del vocabulario, su mero aumento cuantitativo, tiene consecuencias positivas para el sistema lingüístico del individuo, dado que amplía enormemente su capacidad no solo de interpretar, sino también de producir enunciados.

En experiencias prácticas, en especial en lengua inglesa, hay una gran variedad de estudios que ponen de manifiesto como la enseñanza directa de vocabulario contribuye al desarrollo de la lengua (una revisión del tema y el sistema particular de bits de idiomas puede ser visto en Navarro Cordon, 2002). Esta postura se deja ver, en especial, en todos los planes de profesorados de inglés en Argentina, donde en cada una de las materias (incluso en las gramáticas) hay una parte destinada al trabajo con "palabras claves" y "vocabulario específico" de una determinada unidad temática. Esta lógica rige también para los libros de enseñanza del inglés de todos los niveles, que tienen para cada sección un espacio destinado al trabajo intensivo con el vocabulario a través de métodos de enseñanza directa.

En investigaciones científicas, especialmente en el área de desempeño académico, los trabajos de Zapico (2016a, 2016b) ponen de manifiesto una relación en principio correlacional entre el vocabulario léxico-productivo y el desempeño académico en el nivel superior. Si se encontraran correlaciones significativamente estadísticas en futuros 
estudios, esto daría paso directo a la necesidad de desarrollar en las instancias previas al nivel superior (niveles primario y secundario) un desarrollo del léxico-productivo con métodos de enseñanza directa. Incluso con el dato de que existe una correlación entre ambas, el vocabulario no puede seguir siendo dejado de lado en los programas y planificaciones de niveles primario y secundario.

\section{Conclusión}

Durante mucho tiempo la enseñanza de vocabulario de forma directa fue considerada una práctica poco útil y obsoleta, asociada con el modelo tecnocrático en la educación Argentina. No obstante, parece haber sufrido un proceso de arrastre junto con otros conceptos de este modelo (que sí pueden ser considerados obsoletos) y su enseñanza directa fue dejada de lado, en pos de un aprendizaje indirecto o pasivo.

A la luz del fracaso en términos de resultados del enfoque comunicativo en la enseñanza de la lengua, se empiezan a poner en tela de juicio decisiones que se tomaron hace veinte años, tales como: la progresiva desaparición del análisis oracional, la falta de conceptualización en torno a las clases de palabras, la desautorización de la ortografía como elemento estructurante de la escritura aduciendo que obstaculizaba la creatividad, etc. Entre aquellas decisiones estaba la de empezar a reemplazar el aprendizaje directo del vocabulario por un aprendizaje indirecto, a través de una variedad de prácticas de lectura, escritura y oralidad que pasivamente promoverían la incorporación y consolidación del vocabulario en los sujetos.

Además, distintos tipos de estudios, investigaciones, experiencias e incluso opiniones de docentes parecen indicar que el vocabulario es un elemento fundamental en cualquier tipo de aprendizaje, puesto que es la base necesaria para afrontar cualquier tipo de actividad. Dado este carácter central, sumado a las hipótesis positivas sobre su enseñanza directa, se vuelve imprescindible que, progresivamente, se evalúe la posibilidad de implementar de nuevo la enseñanza directa del vocabulario.

Recordemos que enseñar vocabulario no es solo dar palabras. Se trata de ampliar los horizontes de pensamiento de nuestros alumnos, de darles más posibilidades para que digan con más matices qué es lo que les pasa en cada situación, que tengan mayor capacidad de adecuación, que puedan comunicarse mejor. 
En fin, recordar que, como señaló Emerson, "El hombre no es más que la mitad de sí mismo La otra mitad es su expresión”. Por eso mismo, estamos en la obligación de dar a nuestros alumnos muchas más palabras, y que así sean no solo mejores alumnos, sino también mejores seres humanos.

\section{Referencias}

Agustín, M. P. y Barreras, A. (2007) Children's characteristics in vocabulary acquisition and use in the written production. Revista Española de Lingüística Aplicada, 20, 9-26.

Aiello, M. y Grandoli, M. E. (2012). La evaluación de instituciones de educación superior en Argentina: Comparación entre los procesos de evaluación de instituciones universitarias y de institutos de formación docente. Actas de Congreso. Recuperado de http:// www.saece.org.ar/docs/congreso4/trab46.pdf

Barcia, P. (2007). La lengua en los nuevos medios electrónicos. Buenos Aires: AAL.

Boehm, A. E. (1971). Boehm test of basic concepts: forms $A$ and B. New York: The Psychological Corporation.

Carlino, P. (2007). ¿Qué nos dicen las investigaciones internacionales sobre escritura en la universidad? Cuadernos de Psicopedagogía, 4, 21-40.

Carlino, P. (2001). Hacerse cargo de la lectura y la escritura en la enseñanza universitaria de las ciencias sociales y humanas. I Jornadas sobre la lectura y la escritura como prácticas académicas universitarias. Departamento de Educación, Universidad Nacional de Luján, Luján, Prov. de Buenos Aires.

Casso, J. (2010). Análisis y revisión críticas de los materiales de evaluación de la competencia léxica. Elaboración de un test de vocabulario de nivel umbral. Nebrija: Departamento de Lenguas Aplicadas.

Chacón Beltrán, R. (2000). La enseñanza del vocabulario en inglés como L2 el efecto del énfasis en la formación lingüística en el aprendizaje de cognados falsos. Tesis disponible en http://hdl. handle.net/11441/15111

Díaz, G. (2005). Modalidades de enseñanza centradas en el desarrollo de competencias. Orientaciones para obtener el cambio 
metodológico en el ambiente europeo de educación superior. Convocatoria del PROYECTO EA22000055-00111188. España.

Galeote, M., Peraita, H. y Méndez, L. (1997). Programa de intervención en adquisición del léxico en niños de Educación Infantil. Madrid: UNED.

Galve, J. L., García, E. M., y Yuste, J. (1993). CONCEBAS: Test de conceptos básicos para Educación Infantil y Primaria. Madrid: Ed. CEPE.

García-Hoz, V. (1988). Educación personalizada. Madrid: Ediciones RIALP.

García-Hoz, V. (1999). Vocabulario común y su utilización didáctica. Bordón, 51, 141-158.

García, A. M. Bravo, R., Alvero, J., Cuello, R. y Sancho, L. (2005). Competencias y diseño de la evaluación continua y final en el espacio europeo de la educación superior. Catalunya: Ministerio de Educación y Ciencia, Dirección General de Universidades.

Garfias, E (2010) Los jóvenes no leen menos. Periódico La Jornada Domingo 6 de junio de 2010, p. 5. Recuperado de http://www. jornada.unam.mx/2010/06/06/cultura/a05n1cul

Gil, J. (2009). Neurología y lingüística: La teoría de las "redes relacionales" como una alternativa ante Chomsky. Revista de Investigación Lingüistica, 12, 343-374.

Hasbún, L. (2005). The effect of explicit vocabulary teaching on vocabulary acquisition and attitude towards reading. Actualidades Investigativas en Educación, 5, 1-21.

Infobae. (2012). Alarma la cantidad de universitarios que no leen. Recuperado de http://www.infobae.com/2012/11/23/682988-alarma-la-cantidad-universitarios-que-no-entienden-lo-que-leen/

Laco, L., Natalia, L. y Ávila, M. (2010). La lectura y escritura en la formación académica, docente y profesional. Buenos Aires: Editorial de la Universidad Tecnológica Nacional.

Lamb, S. (1999). Senderos del cerebro. Mar del Plata: Eudem.

Lamb, S. (2004a). Dimensions of the Territory of Neurolinguistics. En Language and Reality, London \& New York, Continuum, 16, pp. 318-323.

Lamb, S. (2004b). On the Perception of Speech, capítulo 18 de Language and Reality. 
Marín, M. (2003) Lingüistica y enseñanza de la lengua . Buenos Aires: Grupo Editor Aique

Ministerio de la Nación. (2014). Núcleos de aprendizaje prioritarios. Disponible en www.me.gov.ar/curriform/publica/nap

Mohseni-Far, M. (2008). A cognitively-oriented encapsulation of strategies utilized for lexical development: in search of a flexible and highly interactive curriculum. Porta Linguarum: Revista Internacional de Didáctica de las Lenguas Extranjeras, 9, 35-52.

Moyano, E. (2005). La escritura académica: una tarea interdisciplinaria a lo largo de la currícula universitaria. Textura, 4(4), 109-120.

Navarro Cordon, M. (2002). La enseñanza aprendizaje del vocabulario en inglés y la expresión escrita.

Pleyán, C. (1975). Comprensión y Expresión. Barcelona: Teide.

Requejo, I. (2001). Pobreza de vocabulario y autoría de la palabra y del pensamiento. Recuperado de http://usuarios.arnet.com.ar/yanasu/educacion.htm

Suárez, A., Moreno, J. M. y Godoy, M. J. (2010). Vocabulario y comprensión lectora: Algo más que causa y efecto. Álabe: Revista de Investigación sobre Lectura y Escritura, 1, 0-10.

Ur, P. (1996). A course in language teaching: practice and theory. New York: Cambridge University Press.

Valeiras, N. (2009). El rendimiento académico y la escritura en ingresantes de carreras de ciencias exactas. Revista Educación en Biología. Vol 12, (1).ISBN: 0329-5192.

Vásquez, O., Rey, G., Buubé, C. y Cañibano, A. (2010). Un cambio en la concepción enseñanza-aprendizaje: Formación superior basada en competencias. Memorias del III REPEM. Santa Rosa, La Pampa, Argentina

Yuste, C. y García, N. (1991). Reforzamiento y desarrollo de habilidades básicas. Madrid: ICCE.

Zapico, M. G. (2016a). Evaluación de estrategias de enseñanza orientadas a competencias: una comparación de caso entre estilo orientado a la competencia y el orientado al contenido. Revista Ensayos Pedagógicos, XI(1), 135-146.

Zapico, M. G. (2016b). Evaluación de desempeño académico: la competencia léxica como competencia con validez productiva. Revista Ensayos Pedagógicos, XI(2), 159-171. 